\title{
Bivariate Montgomery identity for alpha diamond integrals
}

\author{
Masud Ahmad' ', Khalid Mahmood Awan², Shehzad Hameed' ${ }^{1}$ Khuram Ali Khan² and \\ Ammara Nosheen ${ }^{1 *}$ (D)
}

"Correspondence:

hammaran@gmail.com

1 Department of Mathematics,

University of Lahore (Sargodha

Campus), Sargodha, Pakistan

Full list of author information is

available at the end of the article

\begin{abstract}
In the paper, some variants of Montgomery identity with the help of delta and nabla integrals are established which are useful to produce Montgomery identity involving alpha diamond integrals for function of two variables. The aforementioned identity is discussed in discrete, continuous, quantum calculus as well and employed to obtain Ostrowski type inequality for monotonically increasing function with respect to both parameters.

MSC: Primary 26D15; secondary 39A13; 34N05

Keywords: Time scales; Montgomery identity; Delta integrals; Nabla integrals; Alpha diamond integrals
\end{abstract}

\section{Introduction}

Mitrinovic, Pecaric, and Fink proved Montgomery identities for functions defined on the real line [17] in the following form:

Let $f:[a, b] \rightarrow \mathbb{R}$ be a differentiable function and $f^{\prime}:[a, b] \rightarrow \mathbb{R}$ be integrable on $[a, b] \in$ $\mathbb{R}$, then

$$
f(x)=\frac{1}{b-a} \int_{a}^{b} f(t) d t+\int_{a}^{b} p(x, t) f^{\prime}(t) d t
$$

where $p(x, t)$ is called Peano kernel, defined in [17]. Some applications of Montgomery identity in the form of inequalities can be found in $[13,15,16]$.

Dragomir et al. extended it for a function of two variables on the real line [12] in the following form:

$$
\begin{aligned}
f(x, y)= & \frac{1}{b-a} \int_{a}^{b} f(t, y) d t+\frac{1}{d-c} \int_{c}^{d} f(x, s) d s \\
& -\frac{1}{(b-a)(d-c)} \int_{a}^{b} \int_{c}^{d} f(t, s) d s d t+\int_{a}^{b} \int_{c}^{d} p(x, t) q(y, s) \frac{\partial^{2} f(t, s)}{\partial t \partial s} d s d t,
\end{aligned}
$$

where $f: I=[a, b] \times[c, d] \rightarrow \mathbb{R}$ is differentiable, the derivative $\frac{\partial^{2} f(t, s)}{\partial t \partial s}$ is integrable on I, $p(x, t)$ and $q(y, s)$ are Peano kernels, defined in [12].

(c) The Author(s) 2019. This article is distributed under the terms of the Creative Commons Attribution 4.0 International License (http://creativecommons.org/licenses/by/4.0/), which permits unrestricted use, distribution, and reproduction in any medium, provided you give appropriate credit to the original author(s) and the source, provide a link to the Creative Commons license, and indicate if changes were made. 
In 1988, Hilger, a German mathematician, presented time scales theory which deals with both discrete and continuous cases simultaneously. Delta and nabla calculus were first two approaches in the theory of time scales. For introduction to the time scales calculus, the readers are referred to $[9,10]$, and some related inequalities can be seen in $[1,3,5,6,8,11$, 14].

Bohner and Matthews proved the following Montgomery identity for functions of one variable by using delta integrals [7].

Let $a, b, s, t \in \mathbb{T} ; a<b$ and $f:[a, b] \rightarrow \mathbb{R}$ be differentiable, then

$$
f(t)=\frac{1}{b-a} \int_{a}^{b} f^{\sigma}(s) \Delta s+\frac{1}{b-a} \int_{a}^{b} p(t, s) f^{\Delta}(s) \Delta s,
$$

where

$$
p(t, s)= \begin{cases}s-a, & a \leq s<t \\ s-b, & t \leq s<b\end{cases}
$$

Remark 1.1 For nabla integrals, (1.3) can be written as follows:

Let $a, b, s, t \in \mathbb{T} ; a<b$ and $f:[a, b] \rightarrow \mathbb{R}$ be differentiable, then

$$
f(t)=\frac{1}{b-a} \int_{a}^{b} f^{\rho}(s) \nabla s+\frac{1}{b-a} \int_{a}^{b} p(t, s) f^{\nabla}(s) \nabla s,
$$

where

$$
p(t, s)= \begin{cases}s-a, & a<s \leq t \\ s-b, & t<s \leq b .\end{cases}
$$

Özkan and Yildrim gave the representation of functions depending on two variables in the form of delta integrals [18]. In 2006, Sheng et al. introduced the combined dynamic derivative, also called alpha diamond dynamic derivative $(\alpha \in[0,1])$, as a linear convex combination of the delta and nabla dynamic derivatives on time scales [19]. The aim of the paper is to extend Montgomery identity by using alpha diamond integrals [2] and to establish respective Ostrowski type inequality for alpha diamond integrals.

\subsection{Preliminaries}

\subsubsection{Alpha diamond derivative [19]}

Definition 1.2 Let $\mathbb{T}$ be a time scale and $f(t)$ be differentiable on $t \in \mathbb{T}$ in delta and nabla senses. For $t \in \mathbb{T}$, we define the alpha diamond derivative $f^{\alpha_{\alpha}}(t)$ by

$$
f^{\diamond}(t)=\alpha f^{\Delta}(t)+(1-\alpha) f^{\nabla}(t) \quad \forall \alpha \in[0,1]
$$

Note that the alpha diamond derivative reduces to the standard delta derivative for $\alpha=1$ and nabla derivative for $\alpha=0$. 


\section{Properties of alpha diamond derivative}

Theorem 1.3 Let $f$ and $g$ be alpha diamond differentiable functions, then:

The sum $f+g: \mathbb{T} \rightarrow \mathbb{R}$ is alpha diamond differentiable at $t \in \mathbb{T}$, satisfying

$$
(f+g)^{\diamond_{\alpha}}(t)=f^{\diamond_{\alpha}}(t)+g^{\diamond_{\alpha}}(t)
$$

The product $f g: \mathbb{T} \rightarrow \mathbb{R}$ is alpha diamond differentiable at $t \in \mathbb{T}$, satisfying

$$
\begin{gathered}
(f g)^{\diamond_{\alpha}}(t)=f^{\diamond_{\alpha}}(t) \cdot g(t)+\alpha f^{\sigma}(t) g^{\Delta}(t)+(1-\alpha) f^{\rho}(t) g^{\nabla}(t) . \\
\text { If } g(t) g^{\sigma}(t) g^{\rho}(t) \neq 0, \frac{f}{g}: \mathbb{T} \rightarrow \mathbb{R} \text { is alpha diamond differentiable at } t \in \mathbb{T}, \text { then } \\
\left(\begin{array}{l}
f \\
g
\end{array}\right)^{\diamond_{\alpha}}(t)=\frac{f^{\diamond_{\alpha}}(t) g^{\sigma}(t) g^{\rho}(t)-\alpha f^{\sigma}(t) g^{\rho}(t) g^{\Delta}(t)-(1-\alpha) f^{\rho}(t) g^{\sigma}(t) g^{\nabla}(t)}{g(t) g^{\sigma}(t) g^{\rho}(t)} .
\end{gathered}
$$

\subsubsection{Alpha diamond integration [2]}

Definition 1.4 Let $a_{1}, a_{2} \in \mathbb{T}$ and $f: \mathbb{T} \rightarrow \mathbb{R}$, then for $\alpha \in[0,1]$, the alpha diamond integral of $f$ is defined by

$$
\int_{a_{1}}^{a_{2}} f(t) \diamond_{\alpha}(t)=\alpha \int_{a_{1}}^{a_{2}} f(t) \Delta t+(1-\alpha) \int_{a_{1}}^{a_{2}} f(t) \nabla t
$$

provided delta and nabla integrals of $f$ exist on $\mathbb{T}$.

Properties of alpha diamond integration

Theorem 1.5 Let $f, g: \mathbb{T} \rightarrow \mathbb{R}$ be alpha diamond integrable on $\left[a_{1}, a_{2}\right]_{\mathbb{T}}$. Let $a_{3} \in\left[a_{1}, a_{2}\right]_{\mathbb{T}}$ with $a_{1}<a_{3}<a_{2}$ and $\lambda \in \mathbb{R}$, then

$$
\begin{aligned}
& \int_{a_{1}}^{a_{1}} f(t) \diamond_{\alpha} t=0 ; \\
& \int_{a_{1}}^{a_{2}} f(t) \diamond_{\alpha} t=\int_{a_{1}}^{a_{3}} f(t) \diamond_{\alpha} t+\int_{a_{3}}^{a_{2}} f(t) \diamond_{\alpha} t ; \\
& \int_{a_{1}}^{a_{2}} f(t) \diamond_{\alpha} t=-\int_{a_{2}}^{a_{1}} f(t) \diamond_{\alpha} t ; \\
& \int_{a_{1}}^{a_{2}}(f+g)(t) \diamond_{\alpha} t=\int_{a_{1}}^{a_{2}} f(t) \diamond_{\alpha}(t)+\int_{a_{1}}^{a_{2}} g(t) \diamond_{\alpha} t ; \\
& \int_{a_{1}}^{a_{2}} \lambda f(t) \diamond_{\alpha} t=\lambda \int_{a_{1}}^{a_{2}} f(t) \diamond_{\alpha} t .
\end{aligned}
$$

The following result can be found in [4] and is used in the proof of next results.

\subsubsection{Fubini's theorem on time scales}

Let $\mathbb{T}_{1}, \mathbb{T}_{2}$ be two time scales. Suppose that $S: \mathbb{T}_{1} \times \mathbb{T}_{2} \rightarrow \mathbb{R}$ is integrable with respect to both time scales. Define $\phi\left(y_{2}\right)=\int_{\mathbb{T}_{1}} S\left(y_{1}, y_{2}\right) \Delta y_{1}$ for a.e. $y_{2} \in \mathbb{T}_{2}$ and $\psi\left(y_{1}\right)=$ 
$\int_{\mathbb{T}_{2}} S\left(y_{1}, y_{2}\right) \Delta y_{2}$ for a.e. $y_{1} \in \mathbb{T}_{1}$. Then $\phi$ and $\psi$ are both differentiable in time scales settings and

$$
\int_{\mathbb{T}_{1}} \Delta y_{1} \int_{\mathbb{T}_{2}} S\left(y_{1}, y_{2}\right) \Delta y_{2}=\int_{\mathbb{T}_{2}} \Delta y_{2} \int_{\mathbb{T}_{1}} S\left(y_{1}, y_{2}\right) \Delta y_{1}
$$

\section{Montgomery identities for function of two variables on time scales}

\subsection{Montgomery identity I}

Lemma 2.1 ([18]) Let $m_{1}, n_{1} \in \mathbb{T}_{1}, m_{2}, n_{2} \in \mathbb{T}_{2}$, and $f \in C C_{\mathrm{rd}}^{1}\left(\left[m_{1}, n_{1}\right]_{\mathbb{T}_{1}} \times\left[m_{2}, n_{2}\right]_{\mathbb{T}_{2}}, \mathbb{R}\right)$, then $\forall(x, y) \in\left[m_{1}, n_{1}\right]_{\mathbb{T}_{1}} \times\left[m_{2}, n_{2}\right]_{\mathbb{T}_{2}}$, we have the representation

$$
\begin{aligned}
f(x, y)= & \frac{1}{k}\left[\int_{m_{1}}^{n_{1}} \int_{m_{2}}^{n_{2}} f\left(\sigma_{1}(s), \sigma_{2}(t)\right) \Delta_{2} t \Delta_{1} s\right. \\
& +\int_{m_{1}}^{n_{1}} \int_{m_{2}}^{n_{2}} q(y, t) \frac{\partial f\left(\sigma_{1}(s), t\right)}{\Delta_{2} t} \Delta_{2} t \Delta_{1} s \\
& +\int_{m_{1}}^{n_{1}} \int_{m_{2}}^{n_{2}} p(x, s) \frac{\partial f\left(s, \sigma_{2}(t)\right)}{\Delta_{1} s} \Delta_{2} t \Delta_{1} s \\
& \left.+\int_{m_{1}}^{n_{1}} \int_{m_{2}}^{n_{2}} p(x, s) q(y, t) \frac{\partial^{2} f(s, t)}{\Delta_{1} s \Delta_{2} t} \Delta_{2} t \Delta_{1} s\right],
\end{aligned}
$$

where $p:\left[m_{1}, n_{1}\right] \times\left[m_{1}, n_{1}\right] \rightarrow \mathbb{R}$ and $q:\left[m_{2}, n_{2}\right] \times\left[m_{2}, n_{2}\right] \rightarrow \mathbb{R}$ are defined as follows:

$$
p(x, s)=\left\{\begin{array}{ll}
s-m_{1}, & \text { if } s \in\left[m_{1}, x\right], \\
s-n_{1}, & \text { if } s \in\left(x, n_{1}\right],
\end{array} \quad q(y, t)= \begin{cases}t-m_{2}, & \text { if } t \in\left[m_{2}, y\right] \\
t-n_{2}, & \text { if } t \in\left(y, n_{2}\right]\end{cases}\right.
$$

and $k=\left(n_{1}-m_{1}\right)\left(n_{2}-m_{2}\right)$.

Note Throughout the paper, $p(x, s), q(y, t)$, and $k$ are as defined in Lemma 2.1.

\subsection{Montgomery identity II}

Lemma 2.2 Let $m_{1}, n_{1} \in \mathbb{T}_{1}, m_{2}, n_{2} \in \mathbb{T}_{2}$, and $f \in C C^{1}\left(\left[m_{1}, n_{1}\right]_{\mathbb{T}_{1}} \times\left[m_{2}, n_{2}\right]_{\mathbb{T}_{2}}, \mathbb{R}\right)$, then $\forall(x, y) \in\left[m_{1}, n_{1}\right]_{\mathbb{T}_{1}} \times\left[m_{2}, n_{2}\right]_{\mathbb{T}_{2}}$, we have the representation

$$
\begin{aligned}
f(x, y)= & \frac{1}{k}\left[\int_{m_{1}}^{n_{1}} \int_{m_{2}}^{n_{2}} f\left(\sigma_{1}(s), \rho_{2}(t)\right) \nabla_{2} t \Delta_{1} s\right. \\
& +\int_{m_{1}}^{n_{1}} \int_{m_{2}}^{n_{2}} p(x, s) f^{\Delta_{1}}\left(s, \rho_{2}(t)\right) \nabla_{2} t \Delta_{1} s \\
& +\int_{m_{1}}^{n_{1}} \int_{m_{2}}^{n_{2}} q(y, t) f^{\nabla_{2}}\left(\sigma_{1}(s), t\right) \nabla_{2} t \Delta_{1} s \\
& \left.+\int_{m_{1}}^{n_{1}} \int_{m_{2}}^{n_{2}} p(x, s) q(y, t) f^{\nabla_{2} \Delta_{1}}(s, t) \nabla_{2} t \Delta_{1} s\right] .
\end{aligned}
$$

Proof Apply $(1.3)$ to $f(\cdot, y)$ for fixed $y \in\left[m_{2}, n_{2}\right]_{\mathbb{T}_{2}}$ to get

$$
f(x, y)=\frac{1}{n_{1}-m_{1}} \int_{m_{1}}^{n_{1}} f\left(\sigma_{1}(s), y\right) \Delta_{1} s+\frac{1}{n_{1}-m_{1}} \int_{m_{1}}^{n_{1}} p(x, s) f^{\Delta_{1}}(s, y) \Delta_{1} s .
$$


Apply (1.4) to $f(s, \cdot)$ for fixed $s \in\left[m_{1}, n_{1}\right]_{\mathbb{T}_{1}}$ to obtain

$$
f(s, y)=\frac{1}{n_{2}-m_{2}} \int_{m_{2}}^{n_{2}} f\left(s, \rho_{2}(t)\right) \nabla_{2} t+\frac{1}{n_{2}-m_{2}} \int_{m_{2}}^{n_{2}} q(y, t) f^{\nabla_{2}}(s, t) \nabla_{2} t .
$$

Again apply (1.4) to $f^{\Delta_{1}}(s, \cdot)$ for $s \in\left[m_{1}, n_{1}\right]_{\mathbb{T}_{1}}$ to get

$$
f^{\Delta_{1}}(s, y)=\frac{1}{n_{2}-m_{2}} \int_{m_{2}}^{n_{2}} f^{\Delta_{1}}\left(s, \rho_{2}(t)\right) \nabla_{2} t+\frac{1}{n_{2}-m_{2}} \int_{m_{2}}^{n_{2}} q(y, t) f^{\nabla_{2} \Delta_{1}}(s, t) \nabla_{2} t .
$$

Substitute (2.4) and (2.5) in (2.3), then use Fubini's theorem to obtain

$$
\begin{aligned}
f(x, y)= & \frac{1}{n_{1}-m_{1}} \int_{m_{1}}^{n_{1}}\left[\frac{1}{n_{2}-m_{2}} \int_{m_{2}}^{n_{2}} f\left(\sigma_{1}(s), \rho_{2}(t)\right) \nabla_{2} t\right. \\
& \left.+\frac{1}{n_{2}-m_{2}} \int_{m_{2}}^{n_{2}} q(y, t) f^{\nabla_{2}}\left(\sigma_{1}(s), t\right) \nabla_{2} t\right] \Delta_{1} s \\
& +\frac{1}{n_{1}-m_{1}} \int_{m_{1}}^{n_{1}} p(x, s)\left[\frac{1}{n_{2}-m_{2}} \int_{m_{2}}^{n_{2}} f^{\Delta_{1}}\left(s, \rho_{2}(t)\right) \nabla_{2} t\right. \\
& \left.+\frac{1}{n_{2}-m_{2}} \int_{m_{2}}^{n_{2}} q(y, t) f^{\nabla_{2} \Delta_{1}}(s, t) \nabla_{2} t\right] \Delta_{1} s,
\end{aligned}
$$

which gives the required result.

\subsection{Montgomery identity III}

Lemma 2.3 Let $m_{1}, n_{1} \in \mathbb{T}_{1}, m_{2}, n_{2} \in \mathbb{T}_{2}$, and $f \in C C^{1}\left(\left[m_{1}, n_{1}\right]_{\mathbb{T}_{1}} \times\left[m_{2}, n_{2}\right]_{\mathbb{T}_{2}}, \mathbb{R}\right)$, then $\forall(x, y) \in\left[m_{1}, n_{1}\right]_{\mathbb{T}_{1}} \times\left[m_{2}, n_{2}\right]_{\mathbb{T}_{2}}$, we have the representation

$$
\begin{aligned}
f(x, y)= & \frac{1}{k}\left[\int_{m_{1}}^{n_{1}} \int_{m_{2}}^{n_{2}} f\left(\rho_{1}(s), \sigma_{2}(t)\right) \Delta_{2} t \nabla_{1} s\right. \\
& +\int_{m_{1}}^{n_{1}} \int_{m_{2}}^{n_{2}} p(x, s) f^{\nabla_{1}}\left(s, \sigma_{2}(t)\right) \Delta_{2} t \nabla_{1} s \\
& +\int_{m_{1}}^{n_{1}} \int_{m_{2}}^{n_{2}} q(y, t) f^{\Delta_{2}}\left(\rho_{1}(s), t\right) \Delta_{2} t \nabla_{1} s \\
& \left.+\int_{m_{1}}^{n_{1}} \int_{m_{2}}^{n_{2}} p(x, s) q(y, t) f^{\nabla_{1} \Delta_{2}}(s, t) \Delta_{2} t \nabla_{1} s\right] .
\end{aligned}
$$

Proof It can be proved accordingly, by shuffling the roles of delta and nabla integrals in Lemma 2.2.

\subsection{Montgomery identity IV}

Lemma 2.4 Let $m_{1}, n_{1} \in \mathbb{T}_{1}, m_{2}, n_{2} \in \mathbb{T}_{2}$, and $f, g \in C C_{\mathrm{ld}}^{1}\left(\left[m_{1}, n_{1}\right]_{\mathbb{T}_{1}} \times\left[m_{2}, n_{2}\right]_{\mathbb{T}_{2}}, \mathbb{R}\right)$, then $\forall(x, y) \in\left[m_{1}, n_{1}\right]_{\mathbb{T}_{1}} \times\left[m_{2}, n_{2}\right]_{\mathbb{T}_{2}}$, we have the representation

$$
\begin{aligned}
f(x, y)= & \frac{1}{k}\left[\int_{m_{1}}^{n_{1}} \int_{m_{2}}^{n_{2}} f\left(\rho_{1}(s), \rho_{2}(t)\right) \nabla_{2} t \nabla_{1} s\right. \\
& +\int_{m_{1}}^{n_{1}} \int_{m_{2}}^{n_{2}} q(y, t) \frac{\partial f\left(\rho_{1}(s), t\right)}{\nabla_{2} t} \nabla_{2} t \nabla_{1} s
\end{aligned}
$$




$$
\begin{aligned}
& +\int_{m_{1}}^{n_{1}} \int_{m_{2}}^{n_{2}} p(x, s) \frac{\partial f\left(s, \rho_{2}(t)\right)}{\nabla_{1} s} \nabla_{2} t \nabla_{1} s \\
& \left.+\int_{m_{1}}^{n_{1}} \int_{m_{2}}^{n_{2}} p(x, s) q(y, t) \frac{\partial^{2} f(s, t)}{\nabla_{1} s \nabla_{2} t} \nabla_{2} t \nabla_{1} s\right] .
\end{aligned}
$$

Proof It can be proved with the help of (1.4) and nabla derivatives and integrals with respect to both variables, instead of (1.3), accordingly as Lemma 2.2.

\subsection{Montgomery identity for alpha diamond integrals}

Theorem 2.5 Let $m_{1}, n_{1} \in \mathbb{T}_{1}, m_{2}, n_{2} \in \mathbb{T}_{2}$, and $f \in C C^{1}\left(\left[m_{1}, n_{1}\right]_{\mathbb{T}_{1}} \times\left[m_{2}, n_{2}\right]_{\mathbb{T}_{2}}, \mathbb{R}\right)$, then $\forall(x, y) \in\left[m_{1}, n_{1}\right]_{\mathbb{T}_{1}} \times\left[m_{2}, n_{2}\right]_{\mathbb{T}_{2}}$, we have

$$
\begin{aligned}
k f(x, y)= & {\left[\alpha_{1} \alpha_{2} \int_{m_{1}}^{n_{1}} \int_{m_{2}}^{n_{2}} f\left(\sigma_{1}(s), \sigma_{2}(t)\right) \Delta_{2} t \Delta_{1} s\right.} \\
& +\alpha_{1}\left(1-\alpha_{2}\right) \int_{m_{1}}^{n_{1}} \int_{m_{2}}^{n_{2}} f\left(\sigma_{1}(s), \rho_{2}(t)\right) \nabla_{2} t \Delta_{1} s \\
& +\alpha_{2}\left(1-\alpha_{1}\right) \int_{m_{1}}^{n_{1}} \int_{m_{2}}^{n_{2}} f\left(\rho_{1}(s), \sigma_{2}(t)\right) \Delta_{2} t \nabla_{1} s \\
& \left.+\left(1-\alpha_{1}\right)\left(1-\alpha_{2}\right) \int_{m_{1}}^{n_{1}} \int_{m_{2}}^{n_{2}} f\left(\rho_{1}(s), \rho_{2}(t)\right) \nabla_{2} t \nabla_{1} s\right] \\
& +\left[\alpha_{2} \int_{m_{1}}^{n_{1}} \int_{m_{2}}^{n_{2}} p(x, s) f^{\diamond_{1}}\left(s, \sigma_{2}(t)\right) \Delta_{2} t \diamond_{\alpha_{1}} s\right. \\
& \left.+\left(1-\alpha_{2}\right) \int_{m_{1}}^{n_{1}} \int_{m_{2}}^{n_{2}} p(x, s) f^{\diamond_{\alpha_{1}}}\left(s, \rho_{2}(t)\right) \nabla_{2} t \diamond_{\alpha_{1}} s\right] \\
& +\left[\alpha_{1} \int_{m_{1}}^{n_{1}} \int_{m_{2}}^{n_{2}} q(y, t) f^{\diamond_{\alpha 2}}\left(\sigma_{1}(s), t\right) \Delta_{2} t \diamond_{\alpha_{1}} s\right. \\
& \left.+\left(1-\alpha_{1}\right) \int_{m_{1}}^{n_{1}} \int_{m_{2}}^{n_{2}} q(y, t) f^{\diamond_{\alpha_{2}}}\left(\rho_{1}(s), t\right) \nabla_{2} t \diamond_{\alpha_{1}} s\right] \\
& +\int_{m_{1}}^{n_{1}} \int_{m_{2}}^{n_{2}} p(x, s) q(y, t) f^{\diamond_{\alpha_{1}} \diamond_{\alpha_{2}}(s, t) \diamond_{\alpha_{2}} t \diamond_{\alpha_{1}} s .}
\end{aligned}
$$

Proof Multiply (2.1) by $\alpha_{1} \alpha_{2},(2.2)$ by $\alpha_{1}\left(1-\alpha_{2}\right),(2.6)$ by $\alpha_{2}\left(1-\alpha_{1}\right)$, and (2.7) by $\left(1-\alpha_{1}\right) \times$ $\left(1-\alpha_{2}\right)$, then add the resultants to obtain

$$
\begin{aligned}
k f(x, y)= & \alpha_{1} \alpha_{2}\left[\int_{m_{1}}^{n_{1}} \int_{m_{2}}^{n_{2}} f\left(\sigma_{1}(s), \sigma_{2}(t)\right) \Delta_{2} t \Delta_{1} s\right. \\
& +\int_{m_{1}}^{n_{1}} \int_{m_{2}}^{n_{2}} p(x, s) f^{\Delta_{1}}\left(s, \sigma_{2}(t)\right) \Delta_{2} t \Delta_{1} s \\
& +\int_{m_{1}}^{n_{1}} \int_{m_{2}}^{n_{2}} q(y, t) f^{\Delta_{2}}\left(\sigma_{1}(s), t\right) \Delta_{2} t \Delta_{1} s \\
& \left.+\int_{m_{1}}^{n_{1}} \int_{m_{2}}^{n_{2}} p(x, s) q(y, t) f^{\Delta_{1} \Delta_{2}}(s, t) \Delta_{2} t \Delta_{1} s\right] \\
& +\alpha_{1}\left(1-\alpha_{2}\right)\left[\int_{m_{1}}^{n_{1}} \int_{m_{2}}^{n_{2}} f\left(\sigma_{1}(s), \rho_{2}(t)\right) \nabla_{2} t \Delta_{1} s\right.
\end{aligned}
$$


Ahmad et al. Advances in Difference Equations

(2019) 2019:314

Page 7 of 13

$$
\begin{aligned}
& +\int_{m_{1}}^{n_{1}} \int_{m_{2}}^{n_{2}} p(x, s) f^{\Delta_{1}}\left(s, \rho_{2}(t)\right) \nabla_{2} t \Delta_{1} s \\
& +\int_{m_{1}}^{n_{1}} \int_{m_{2}}^{n_{2}} q(y, t) f^{\nabla_{2}}\left(\sigma_{1}(s), t\right) \nabla_{2} t \Delta_{1} s \\
& \left.+\int_{m_{1}}^{n_{1}} \int_{m_{2}}^{n_{2}} p(x, s) q(y, t) f^{\Delta_{1} \nabla_{2}}(s, t) \nabla_{2} t \Delta_{1} s\right] \\
& +\alpha_{2}\left(1-\alpha_{1}\right)\left[\int_{m_{1}}^{n_{1}} \int_{m_{2}}^{n_{2}} f\left(\rho_{1}(s), \sigma_{2}(t)\right) \Delta_{2} t \nabla_{1} s\right] \\
& +\int_{m_{1}}^{n_{1}} \int_{m_{2}}^{n_{2}} p(x, s) f^{\nabla_{1}}\left(s, \sigma_{2}(t)\right) \Delta_{2} t \nabla_{1} s \\
& +\int_{m_{1}}^{n_{1}} \int_{m_{2}}^{n_{2}} q(y, t) f^{\Delta_{2}}\left(\rho_{1}(s), t\right) \Delta_{2} t \nabla_{1} s \\
& +\int_{m_{1}}^{n_{1}} \int_{m_{2}}^{n_{2}} p(x, s) q(y, t) f^{\nabla_{1} \Delta_{2}}(s, t) \Delta_{2} t \nabla_{1} s \\
& +\left(1-\alpha_{1}\right)\left(1-\alpha_{2}\right)\left[\int_{m_{1}}^{n_{1}} \int_{m_{2}}^{n_{2}} f\left(\rho_{1}(s), \rho_{2}(t)\right) \nabla_{2} t \nabla_{1} s\right. \\
& +\int_{m_{1}}^{n_{1}} \int_{m_{2}}^{n_{2}} p(x, s) f^{\nabla_{1}}\left(s, \rho_{2}(t)\right) \nabla_{2} t \nabla_{1} s \\
& +\int_{m_{1}}^{n_{1}} \int_{m_{2}}^{n_{2}} q(y, t) f^{\nabla_{2}}\left(\rho_{1}(s), t\right) \nabla_{2} t \nabla_{1} s \\
& \left.+\int_{m_{1}}^{n_{1}} \int_{m_{2}}^{n_{2}} p(x, s) q(y, t) f^{\nabla_{1} \nabla_{2}}(s, t) \nabla_{2} t \nabla_{1} s\right] \\
& =\left[\alpha_{1} \alpha_{2} \int_{m_{1}}^{n_{1}} \int_{m_{2}}^{n_{2}} f\left(\sigma_{1}(s), \sigma_{2}(t)\right) \Delta_{2} t \Delta_{1} s\right. \\
& +\alpha_{1}\left(1-\alpha_{2}\right) \int_{m_{1}}^{n_{1}} \int_{m_{2}}^{n_{2}} f\left(\sigma_{1}(s), \rho_{2}(t)\right) \nabla_{2} t \Delta_{1} s \\
& +\alpha_{2}\left(1-\alpha_{1}\right) \int_{m_{1}}^{n_{1}} \int_{m_{2}}^{n_{2}} f\left(\rho_{1}(s), \sigma_{2}(t)\right) \Delta_{2} t \nabla_{1} s \\
& \left.+\left(1-\alpha_{1}\right)\left(1-\alpha_{2}\right) \int_{m_{1}}^{n_{1}} \int_{m_{2}}^{n_{2}} f\left(\rho_{1}(s), \rho_{2}(t)\right) \nabla_{2} t \nabla_{1} s\right] \\
& +\left[\alpha_{1} \alpha_{2} \int_{m_{1}}^{n_{1}} \int_{m_{2}}^{n_{2}} p(x, s) f^{\Delta_{1}}\left(s, \sigma_{2}(t)\right) \Delta_{2} t \Delta_{1} s\right. \\
& +\alpha_{1}\left(1-\alpha_{2}\right) \int_{m_{1}}^{n_{1}} \int_{m_{2}}^{n_{2}} p(x, s) f^{\Delta_{1}}\left(s, \rho_{2}(t)\right) \nabla_{2} t \Delta_{1} s \\
& +\alpha_{2}\left(1-\alpha_{1}\right) \int_{m_{1}}^{n_{1}} \int_{m_{2}}^{n_{2}} p(x, s) f^{\nabla_{1}}\left(s, \sigma_{2}(t)\right) \Delta_{2} t \nabla_{1} s \\
& \left.+\left(1-\alpha_{1}\right)\left(1-\alpha_{2}\right) \int_{m_{1}}^{n_{1}} \int_{m_{2}}^{n_{2}} p(x, s) f^{\nabla_{1}}\left(s, \rho_{2}(t)\right) \nabla_{2} t \nabla_{1} s\right] \\
& +\left[\alpha_{1} \alpha_{2} \int_{m_{1}}^{n_{1}} \int_{m_{2}}^{n_{2}} q(y, t) f^{\Delta_{2}}\left(\sigma_{1}(s), t\right) \Delta_{2} t \Delta_{1} s\right. \\
& +\alpha_{1}\left(1-\alpha_{2}\right) \int_{m_{1}}^{n_{1}} \int_{m_{2}}^{n_{2}} q(y, t) f^{\nabla_{2}}\left(\sigma_{1}(s), t\right) \nabla_{2} t \Delta_{1} s
\end{aligned}
$$




$$
\begin{aligned}
& +\alpha_{2}\left(1-\alpha_{1}\right) \int_{m_{1}}^{n_{1}} \int_{m_{2}}^{n_{2}} q(y, t) f^{\Delta_{2}}\left(\rho_{1}(s), t\right) \Delta_{2} t \nabla_{1} s \\
& \left.+\left(1-\alpha_{1}\right)\left(1-\alpha_{2}\right) \int_{m_{1}}^{n_{1}} \int_{m_{2}}^{n_{2}} q(y, t) f^{\nabla_{2}}\left(\rho_{1}(s), t\right) \nabla_{2} t \nabla_{1} s\right] \\
& +\left[\alpha_{1} \alpha_{2} \int_{m_{1}}^{n_{1}} \int_{m_{2}}^{n_{2}} p(x, s) q(y, t) f^{\Delta_{1} \Delta_{2}}(s, t) \Delta_{2} t \Delta_{1} s\right. \\
& +\alpha_{1}\left(1-\alpha_{2}\right) \int_{m_{1}}^{n_{1}} \int_{m_{2}}^{n_{2}} p(x, s) q(y, t) f^{\Delta_{1} \nabla_{2}}(s, t) \nabla_{2} t \Delta_{1} s \\
& +\alpha_{2}\left(1-\alpha_{1}\right) \int_{m_{1}}^{n_{1}} \int_{m_{2}}^{n_{2}} p(x, s) q(y, t) f^{\nabla_{1} \Delta_{2}}(s, t) \Delta_{2} t \nabla_{1} s \\
& \left.+\left(1-\alpha_{1}\right)\left(1-\alpha_{2}\right) \int_{m_{1}}^{n_{1}} \int_{m_{2}}^{n_{2}} p(x, s) q(y, t) f^{\nabla_{1} \nabla_{2}}(s, t) \nabla_{2} t \nabla_{1} s\right],
\end{aligned}
$$

and after simplification one gets the required result.

Example 2.6 For $\mathbb{T}_{1}=h_{1} \mathbb{N}, \mathbb{T}_{2}=h_{2} \mathbb{N}, h_{1}, h_{2}>0$, (2.8) can be written as follows:

$$
\begin{aligned}
& k f(x, y)=\alpha_{1} \alpha_{2} \sum_{i=\frac{m_{1}}{h_{1}}}^{\frac{n_{1}}{h_{1}}-1} \sum_{j=\frac{m_{2}}{h_{2}}}^{\frac{h_{2}}{h_{2}}-1} f\left((i+1) h_{1},(i+1) h_{2}\right) h_{1} h_{2} \\
& +\alpha_{1}\left(1-\alpha_{2}\right) \sum_{i=\frac{m_{1}}{h_{1}}}^{\frac{n_{1}}{h_{1}}-1} \sum_{j=\frac{m_{2}}{h_{2}}+1}^{\frac{n_{2}}{h_{2}}} f\left((i+1) h_{1},(i-1) h_{2}\right) h_{1} h_{2} \\
& +\alpha_{2}\left(1-\alpha_{1}\right) \sum_{i=\frac{m_{1}}{h_{1}}+1}^{\frac{n_{1}}{h_{1}}} \sum_{j=\frac{m_{2}}{h_{2}}}^{\frac{n_{2}}{h_{2}}-1} f\left((i-1) h_{1},(i+1) h_{2}\right) h_{1} h_{2} \\
& +\left(1-\alpha_{1}\right)\left(1-\alpha_{2}\right) \sum_{i=\frac{m_{1}}{h_{1}}+1}^{\frac{n_{1}}{h_{1}}} \sum_{j=\frac{m_{2}}{h_{2}}+1}^{\frac{n_{2}}{h_{2}}} f\left((i-1) h_{1},(i-1) h_{2}\right) h_{1} h_{2} \\
& +\alpha_{2}\left[\alpha_{1} \sum_{i=\frac{m_{1}}{h_{1}}}^{\frac{n_{1}}{h_{1}}-1} \sum_{j=\frac{m_{2}}{h_{2}}}^{\frac{n_{2}}{h_{2}}-1} p(x, i+1) f^{\diamond \alpha_{1}}\left((i+1) h_{1},(i+1) h_{2}\right) h_{1} h_{2}\right. \\
& \left.+\left(1-\alpha_{1}\right) \sum_{i=\frac{m_{1}}{h_{1}}+1}^{\frac{n_{1}}{h_{1}}} \sum_{j=\frac{m_{2}}{h_{2}}}^{\frac{n_{2}}{h_{2}}-1} p(x, i+1) f^{\diamond \alpha_{1}}\left((i-1) h_{1},(i+1) h_{2}\right) h_{1} h_{2}\right] \\
& +\left(1-\alpha_{2}\right)\left[\alpha_{1} \sum_{i=\frac{m_{1}}{h_{1}}}^{\frac{n_{1}}{h_{1}}-1} \sum_{j=\frac{m_{2}}{h_{2}}+1}^{\frac{n_{2}}{h_{2}}} p(x, i-1) f^{\diamond \alpha_{1}}\left((i+1) h_{1},(i-1) h_{2}\right) h_{1} h_{2}\right. \\
& \left.+\left(1-\alpha_{1}\right) \sum_{i=\frac{m_{1}}{h_{1}}+1}^{\frac{n_{1}}{h_{1}}} \sum_{j=\frac{m_{2}}{h_{2}}+1}^{\frac{n_{2}}{h_{2}}} p(x, i-1) f^{\diamond \alpha_{1}}\left((i-1) h_{1},(i-1) h_{2}\right) h_{1} h_{2}\right]
\end{aligned}
$$




$$
\begin{aligned}
& +\alpha_{1}\left[\alpha_{2} \sum_{i=\frac{m_{1}}{h_{1}}}^{\frac{h_{1}}{h_{1}}-1} \sum_{j=\frac{m_{2}}{h_{2}}}^{\frac{h_{2}}{h_{2}}-1} q(y, i+1) f^{\diamond \alpha_{2}}\left((i+1) h_{1},(i+1) h_{2}\right) h_{1} h_{2}\right. \\
& \left.+\left(1-\alpha_{2}\right) \sum_{i=\frac{m_{1}}{h_{1}}}^{\substack{n_{1} \\
h_{1}}} \sum_{j=\frac{m_{2}}{h_{2}}+1}^{\substack{h_{2} \\
h_{2}}} q(y, i+1) f^{\diamond \alpha_{2}}\left((i+1) h_{1},(i-1) h_{2}\right) h_{1} h_{2}\right] \\
& +\left(1-\alpha_{1}\right)\left[\alpha_{2} \sum_{i=\frac{m_{1}}{h_{1}}+1}^{\frac{n_{1}}{h_{1}}} \sum_{j=\frac{m_{2}}{h_{2}}}^{\frac{n_{2}}{h_{2}}-1} q(y, i-1) f^{\diamond \alpha_{2}}\left((i-1) h_{1},(i+1) h_{2}\right) h_{1} h_{2}\right. \\
& \left.+\left(1-\alpha_{2}\right) \sum_{i=\frac{m_{1}}{h_{1}}+1}^{\frac{n_{1}}{h_{1}}} \sum_{j=\frac{m_{2}}{h_{2}}+1}^{\frac{n_{2}}{h_{2}}} q(y, i-1) f^{\diamond \alpha_{2}}\left((i-1) h_{1},(i-1) h_{2}\right) h_{1} h_{2}\right] \\
& +\alpha_{1} \alpha_{2} \sum_{i=\frac{m_{1}}{h_{1}}}^{\frac{h_{1}}{h_{1}}-1} \sum_{j=\frac{m_{2}}{h_{2}}}^{\frac{n_{2}}{h_{2}}-1} p(x, i+1) q(y, i+1) f^{\diamond \alpha_{1} \diamond \alpha_{2}}\left((i+1) h_{1},(i+1) h_{2}\right) h_{1} h_{2} \\
& +\alpha_{1}\left(1-\alpha_{2}\right) \sum_{i=\frac{m_{1}}{h_{1}}}^{\frac{n_{1}}{h_{1}}-1} \sum_{j=\frac{m_{2}}{h_{2}}+1}^{\frac{n_{2}}{h_{2}}} p(x, i+1) q(y, i-1) f^{\diamond \alpha_{1} \diamond \alpha_{2}}\left((i+1) h_{1},(i-1) h_{2}\right) h_{1} h_{2} \\
& +\alpha_{2}\left(1-\alpha_{1}\right) \sum_{i=\frac{m_{1}}{h_{1}}+1}^{\frac{n_{1}}{h_{1}}} \sum_{j=\frac{m_{2}}{h_{2}}}^{\frac{n_{2}}{h_{2}}-1} p(x, i-1) q(y, i+1) f^{\diamond \alpha_{1} \diamond \alpha_{2}}\left((i-1) h_{1},(i+1) h_{2}\right) h_{1} h_{2} \\
& +\left(1-\alpha_{1}\right)\left(1-\alpha_{2}\right) \sum_{i=\frac{m_{1}}{h_{1}}+1}^{\frac{n_{1}}{h_{1}}} \sum_{j=\frac{m_{2}}{h_{2}}+1}^{\frac{n_{2}}{h_{2}}} p(x, i-1) q(y, i-1) \\
& \times f^{\diamond \alpha_{1} \diamond \alpha_{2}}\left((i-1) h_{1},(i-1) h_{2}\right) h_{1} h_{2} \text {. }
\end{aligned}
$$

Example 2.7 If $\mathbb{T}_{1}=q_{1}^{\mathbb{N}}, \mathbb{T}_{2}=q_{2}^{\mathbb{N}}, q_{1}, q_{2}>1$, and $a_{1}=q_{1}^{m_{1}}, b_{1}=q_{1}^{n_{1}}, c_{1}=q_{2}^{m_{2}}$, and $d_{1}=q_{2}^{n_{2}}$, then for $m_{1}, m_{2}, n_{1}, n_{2} \in \mathbb{N}$, (2.8) takes the form

$$
\begin{aligned}
& \left(q_{1}^{n_{1}}-q_{1}^{m_{1}}\right)\left(q_{2}^{n_{2}}-q_{2}^{m_{2}}\right) f(x, y) \\
& =\left(q_{1}-1\right)\left(q_{2}-1\right)\left[\alpha_{1} \alpha_{2} \sum_{k_{1}=m_{1}}^{n_{1}-1} \sum_{k_{2}=m_{2}}^{n_{2}-1} f\left(q_{1}^{k_{1}+1}, q_{2}^{k_{2}+1}\right) q_{1}^{k_{1}} q_{2}^{k_{2}}\right. \\
& \quad+\alpha_{1}\left(1-\alpha_{2}\right) \sum_{k_{1}=m_{1}}^{n_{1}-1} \sum_{k_{2}=m_{2}+1}^{n_{2}} f\left(q_{1}^{k_{1}+1}, q_{2}^{k_{2}-1}\right) q_{1}^{k_{1}} q_{2}^{k_{2}-1} \\
& \quad+\alpha_{2}\left(1-\alpha_{1}\right) \sum_{k_{1}=m_{1}+1}^{n_{1}} \sum_{k_{2}=m_{2}}^{n_{2}-1} f\left(q_{1}^{k_{1}-1}, q_{2}^{k_{2}+1}\right) q_{1}^{k_{1}-1} q_{2}^{k_{2}} \\
& \quad+\left(1-\alpha_{1}\right)\left(1-\alpha_{2}\right) \sum_{k_{1}=m_{1}+1}^{n_{1}} \sum_{k_{2}=m_{2}+1}^{n_{2}} f\left(q_{1}^{k_{1}-1}, q_{2}^{k_{2}-1}\right) q_{1}^{k_{1}-1} q_{2}^{k_{2}-1}
\end{aligned}
$$




$$
\begin{aligned}
& +\alpha_{2}\left\{\alpha_{1} \sum_{k_{1}=m_{1}}^{n_{1}-1} \sum_{k_{2}=m_{2}}^{n_{2}-1} p\left(x, q_{2}^{k_{2}+1}\right) f^{\diamond \alpha_{1}}\left(q_{1}^{k_{1}+1}, q_{2}^{k_{2}+1}\right) q_{1}^{k_{1}} q_{2}^{k_{2}}\right. \\
& \left.+\left(1-\alpha_{1}\right) \sum_{k_{1}=m_{1}+1}^{n_{1}} \sum_{k_{2}=m_{2}}^{n_{2}-1} p\left(x, q_{2}^{k_{2}+1}\right) f^{\diamond \alpha_{1}}\left(q_{1}^{k_{1}-1}, q_{2}^{k_{2}+1}\right) q_{1}^{k_{1}-1} q_{2}^{k_{2}}\right\} \\
& +\left(1-\alpha_{2}\right)\left\{\alpha_{1} \sum_{k_{1}=m_{1}}^{n_{1}-1} \sum_{k_{2}=m_{2}+1}^{n_{2}} p\left(x, q_{2}^{k_{2}-1}\right) f^{\diamond \alpha_{1}}\left(q_{1}^{k_{1}+1}, q_{2}^{k_{2}-1}\right) q_{1}^{k_{1}} q_{2}^{k_{2}-1}\right. \\
& \left.+\left(1-\alpha_{1}\right) \sum_{k_{1}=m_{1}+1}^{n_{1}} \sum_{k_{2}=m_{2}+1}^{n_{2}} p\left(x, q_{2}^{k_{2}-1}\right) f^{\diamond \alpha_{1}}\left(q_{1}^{k_{1}-1}, q_{2}^{k_{2}-1}\right) q_{1}^{k_{1}-1} q_{2}^{k_{2}-1}\right\} \\
& +\alpha_{1}\left\{\alpha_{2} \sum_{k_{1}=m_{1}}^{n_{1}-1} \sum_{k_{2}=m_{2}}^{n_{2}-1} q\left(y, q_{2}^{k_{2}+1}\right) f^{\diamond \alpha_{2}}\left(q_{1}^{k_{1}+1}, q_{2}^{k_{2}+1}\right) q_{1}^{k_{1}} q_{2}^{k_{2}}\right. \\
& \left.+\left(1-\alpha_{2}\right) \sum_{k_{1}=m_{1}}^{n_{1}-1} \sum_{k_{2}=m_{2}+1}^{n_{2}} q\left(y, q_{2}^{k_{2}+1}\right) f^{\diamond \alpha_{2}}\left(q_{1}^{k_{1}+1}, q_{2}^{k_{2}-1}\right) q_{1}^{k_{1}} q_{2}^{k_{2}-1}\right\} \\
& +\left(1-\alpha_{1}\right)\left\{\alpha_{2} \sum_{k_{1}=m_{1}+1}^{n_{1}} \sum_{k_{2}=m_{2}}^{n_{2}-1} q\left(y, q_{2}^{k_{2}-1}\right) f^{\diamond \alpha_{2}}\left(q_{1}^{k_{1}-1}, q_{2}^{k_{2}+1}\right) q_{1}^{k_{1}-1} q_{2}^{k_{2}}\right. \\
& \left.+\left(1-\alpha_{2}\right) \sum_{k_{1}=m_{1}+1}^{n_{1}} \sum_{k_{2}=m_{2}+1}^{n_{2}} q\left(y, q_{2}^{k_{2}-1}\right) f^{\diamond \alpha_{2}}\left(q_{1}^{k_{1}-1}, q_{2}^{k_{2}-1}\right) q_{1}^{k_{1}-1} q_{2}^{k_{2}-1}\right\} \\
& +\alpha_{1} \alpha_{2} \sum_{k_{1}=m_{1}}^{n_{1}-1} \sum_{k_{2}=m_{2}}^{n_{2}-1} p\left(x, q_{1}^{k_{1}+1}\right) q\left(y, q_{2}^{k_{2}+1}\right) f^{\diamond \alpha_{1} \diamond \alpha_{2}}\left(q_{1}^{k_{1}+1}, q_{2}^{k_{2}+1}\right) q_{1}^{k_{1}} q_{2}^{k_{2}} \\
& \left.+\alpha_{1}\left(1-\alpha_{2}\right) \sum_{k_{1}=m_{1}}^{n_{1}-1} \sum_{k_{2}=m_{2}+1}^{n_{2}} p\left(x, q_{1}^{k_{1}+1}\right) q\left(y, q_{2}^{k_{2}-1}\right)\right) f^{\diamond \alpha_{1} \diamond \alpha_{2}}\left(q_{1}^{k_{1}+1}, q_{2}^{k_{2}-1}\right) q_{1}^{k_{1}} q_{2}^{k_{2}-1} \\
& +\alpha_{2}\left(1-\alpha_{1}\right) \sum_{k_{1}=m_{1}+1}^{n_{1}} \sum_{k_{2}=m_{2}}^{n_{2}-1} p\left(x, q_{1}^{k_{1}-1}\right) q\left(y, q_{2}^{k_{2}+1}\right) f^{\diamond \alpha_{1} \diamond \alpha_{2}}\left(q_{1}^{k_{1}-1}, q_{2}^{k_{2}+1}\right) q_{1}^{k_{1}-1} q_{2}^{k_{2}} \\
& \left.+\left(1-\alpha_{1}\right)\left(1-\alpha_{2}\right) \sum_{k_{1}=m_{1}+1}^{n_{1}} \sum_{k_{2}=m_{2}+1}^{n_{2}} p\left(x, q_{1}^{k_{1}-1}\right) q\left(y, q_{2}^{k_{2}-1}\right) f^{\diamond \alpha_{1} \diamond \alpha_{2}}\left(q_{1}^{k_{1}-1}, q_{2}^{k_{2}-1}\right)\right] .
\end{aligned}
$$

Remark 2.8 For $\mathbb{T}_{1}=\mathbb{T}_{2}=\mathbb{R},(2.8)$ coincides with (1.2).

Corollary 2.9 In addition to the conditions of Theorem 2.5, if the function is monotonically increasing, then we have the following inequality:

$$
\begin{aligned}
f(x, y) \leq & \frac{1}{k}\left[\int_{m_{1}}^{n_{1}} \int_{m_{2}}^{n_{2}} f\left(\sigma_{1}(s), \sigma_{2}(t)\right) \diamond_{\alpha_{2}} t \diamond_{\alpha_{1}} s\right. \\
& +\int_{m_{1}}^{n_{1}} \int_{m_{2}}^{n_{2}} p(x, s) f^{\diamond_{\alpha_{1}}}\left(s, \sigma_{2}(t)\right) \diamond_{\alpha_{2}} t \diamond_{\alpha_{1}} s \\
& +\int_{m_{1}}^{n_{1}} \int_{m_{2}}^{n_{2}} q(y, t) f^{\diamond_{\alpha_{2}}}\left(\sigma_{1}(s), t\right) \diamond_{\alpha_{2}} t \diamond_{\alpha_{1}} s \\
& +\int_{m_{1}}^{n_{1}} \int_{m_{2}}^{n_{2}} p(x, s) q(y, t) f^{\left.\diamond_{\alpha_{2}} \diamond_{\alpha_{1}}(s, t) \diamond_{\alpha_{2}} t \diamond_{\alpha_{1}} s\right] .}
\end{aligned}
$$


Proof Since $f$ is increasing, $\rho_{i}\left(t_{i}\right) \leq t_{i} \leq \sigma_{i}\left(t_{i}\right), \forall t_{i} \in \mathbb{T}_{i}$, therefore by replacing $\rho_{i}$ with $\sigma_{i}$, on the right-hand side of (2.8), one gets the required result.

\section{Ostrowski type inequality}

Theorem 3.1 Let $m_{1}, n_{1} \in \mathbb{T}_{1}, m_{2}, n_{2} \in \mathbb{T}_{2}, f \in C C^{1}\left(\left[m_{1}, n_{1}\right]_{\mathbb{T}_{1}} \times\left[m_{2}, n_{2}\right]_{\mathbb{T}_{2}}, \mathbb{R}\right)$, further assume $f(\cdot, \cdot)$ is an increasing function with respect to both parameters, then $\forall(x, y) \in$ $\left[m_{1}, n_{1}\right]_{\mathbb{T}_{1}} \times\left[m_{2}, n_{2}\right]_{\mathbb{T}_{2}}$, one gets

$$
\begin{aligned}
\mid f(x, y) & -\frac{1}{k} \int_{m_{1}}^{n_{1}} \int_{m_{2}}^{n_{2}} f\left(\sigma_{1}(s), \sigma_{2}(t)\right) \diamond_{\alpha_{2}} t \diamond_{\alpha_{1}} s \mid \\
\leq & \frac{1}{\left(n_{1}-m_{1}\right)} M_{1}\left[\hat{h_{2}}\left(x, m_{1}\right)-\hat{h_{2}}\left(x, n_{1}\right)\right]+\frac{1}{\left(n_{2}-m_{2}\right)} M_{2}\left[\hat{h_{2}}\left(y, m_{2}\right)-\hat{h_{2}}\left(y, n_{2}\right)\right] \\
& +\frac{1}{k} M_{3}\left[\hat{h_{2}}\left(x, m_{1}\right)-\hat{h_{2}}\left(x, n_{1}\right)\right]\left[\hat{h_{2}}\left(y, m_{2}\right)-\hat{h_{2}}\left(y, n_{2}\right)\right],
\end{aligned}
$$

where

$$
\begin{array}{ll}
\hat{h_{2}}\left(x, m_{1}\right)=\int_{m_{1}}^{x}\left(s-m_{1}\right) \diamond_{\alpha_{1}} s, & \hat{h_{2}}\left(x, n_{1}\right)=\int_{n_{1}}^{x}\left(s-n_{1}\right) \diamond_{\alpha_{1}} s, \\
\hat{h_{2}}\left(y, m_{2}\right)=\int_{m_{2}}^{y}\left(t-m_{2}\right) \diamond_{\alpha_{2}} t, & \hat{h_{2}}\left(y, n_{2}\right)=\int_{n_{2}}^{y}\left(t-n_{2}\right) \diamond_{\alpha_{2}} t .
\end{array}
$$

Proof Inequality (2.9) can be written as

$$
\begin{aligned}
f(x, y) & -\frac{1}{k} \int_{m_{1}}^{n_{1}} \int_{m_{2}}^{n_{2}} f\left(\sigma_{1}(s), \sigma_{2}(t)\right) \diamond_{\alpha_{2}} t \diamond_{\alpha_{1}} s \\
\leq & \frac{1}{k}\left[\int_{m_{1}}^{n_{1}} \int_{m_{2}}^{n_{2}} p(x, s) f^{\diamond_{\alpha_{1}}}\left(s, \sigma_{2}(t)\right) \diamond_{\alpha_{2}} t \diamond_{\alpha_{1}} s\right. \\
& +\int_{m_{1}}^{n_{1}} \int_{m_{2}}^{n_{2}} q(y, t) f^{\diamond_{\alpha_{2}}}\left(\sigma_{1}(s), t\right) \diamond_{\alpha_{2}} t \diamond_{\alpha_{1}} s \\
& \left.+\int_{m_{1}}^{n_{1}} \int_{m_{2}}^{n_{2}} p(x, s) q(y, t) f^{\diamond_{\alpha_{2}} \diamond_{\alpha_{1}} s}(s, t) \diamond_{\alpha_{2}} t \diamond_{\alpha_{1}} s\right] .
\end{aligned}
$$

By taking absolute value on both sides, one gets

$$
\begin{aligned}
& \mid f(x, y)-\frac{1}{k} \int_{m_{1}}^{n_{1}} \int_{m_{2}}^{n_{2}} f\left(\sigma_{1}(s), \sigma_{2}(t)\right) \diamond_{\alpha_{2}} t \diamond_{\alpha_{1}} s \mid \\
& \leq \frac{1}{k}\left[M_{1}\left|\int_{m_{1}}^{n_{1}} \int_{m_{2}}^{n_{2}} p(x, s) \diamond_{\alpha_{2}} t \diamond_{\alpha_{1}} s\right|+M_{2}\left|\int_{m_{1}}^{n_{1}} \int_{m_{2}}^{n_{2}} q(y, t) \diamond_{\alpha_{2}} t \diamond_{\alpha_{1}} s\right|\right. \\
&\left.\quad+M_{3}\left|\int_{m_{1}}^{n_{1}} \int_{m_{2}}^{n_{2}} p(x, s) q(y, t) \diamond_{\alpha_{2}} t \diamond_{\alpha_{1}} s\right|\right]
\end{aligned}
$$

where

$$
\begin{aligned}
& M_{1}=\operatorname{Sup}_{m_{1}<s<n_{1}}\left|f^{\diamond \alpha_{1}}\left(s, \sigma_{2}(t)\right)\right|, \quad M_{2}=\operatorname{Sup}_{m_{2}<t<n_{2}}\left|f^{\diamond \alpha_{2}}\left(\sigma_{1}(s), t\right)\right| \quad \text { and } \\
& M_{3}=\operatorname{Sup}_{m_{1}<s<n_{1}, m_{2}<t<n_{2}}\left|f^{\diamond \alpha_{1} \diamond \alpha_{2}}(s, t)\right|,
\end{aligned}
$$


which gives

$$
\begin{aligned}
\mid f(x, y) & -\frac{1}{k} \int_{m_{1}}^{n_{1}} \int_{m_{2}}^{n_{2}} f\left(\sigma_{1}(s), \sigma_{2}(t)\right) \diamond_{\alpha_{2}} t \diamond_{\alpha_{1}} s \mid \\
\leq & \frac{1}{k}\left[M_{1}\left(n_{2}-m_{2}\right) \int_{m_{1}}^{n_{1}} p(x, s) \diamond_{\alpha_{1}} s+M_{2}\left(n_{1}-m_{1}\right) \int_{m_{2}}^{n_{2}} q(y, t) \diamond_{\alpha_{2}} t\right. \\
& \left.+M_{3} \int_{m_{1}}^{n_{1}} p(x, s) \diamond_{\alpha_{1}} s \int_{m_{2}}^{n_{2}} q(y, t) \diamond_{\alpha_{2}} t\right] \\
= & \frac{1}{\left(n_{1}-m_{1}\right)} M_{1}\left[\hat{h_{2}}\left(x, m_{1}\right)-\hat{h_{2}}\left(x, n_{1}\right)\right]+\frac{1}{\left(n_{2}-m_{2}\right)} M_{2}\left[\hat{h_{2}}\left(y, m_{2}\right)-\hat{h_{2}}\left(y, n_{2}\right)\right] \\
& +\frac{1}{k} M_{3}\left[\hat{h_{2}}\left(x, m_{1}\right)-\hat{h_{2}}\left(x, n_{1}\right)\right]\left[\hat{h_{2}}\left(y, m_{2}\right)-\hat{h_{2}}\left(y, n_{2}\right)\right],
\end{aligned}
$$

which is the required result.

\section{Acknowledgements}

The authors are thankful to the editor and referees for their fruitful time to read and make valuable suggestions to the article.

\section{Funding}

Not applicable.

\section{Availability of data and materials}

The datasets used or analysed during the current study are cited in references and are available from the corresponding author on reasonable request.

\section{Competing interests}

The authors declare that they have no competing interests.

\section{Authors' contributions}

All the authors equally participated to complete this research article. They read and approved the final manuscript.

\section{Author details}

${ }^{1}$ Department of Mathematics, University of Lahore (Sargodha Campus), Sargodha, Pakistan. ${ }^{2}$ Department of Mathematics, University of Sargodha, Sargodha, Pakistan.

\section{Publisher's Note}

Springer Nature remains neutral with regard to jurisdictional claims in published maps and institutional affiliations.

Received: 9 May 2019 Accepted: 23 July 2019 Published online: 02 August 2019

\section{References}

1. Ahmad, W., Khan, K.A., Nosheen, A., Sultan, M.A.: Copson-Leindler type inequalities for function of several variables on time scales. Punjab Univ. J. Math. 51(8), 157-168 (2019)

2. Ammi, M.R.S., Ferreira, R.A.C., Torres, D.F.M.: Diamond-alpha integral inequalities on time scales. Int. J. Math. Stat. 5(A09), 52-59 (2009)

3. Asraf, M.S., Khan, K.A., Nosheen, A.: Hardy-Copson type inequalities on time scales for the functions of ' $n$ ' independent variables. Int. J. Anal. Appl. 17(2), 244-259 (2019). https://doi.org/10.28924/2291-8639-17-2019-244

4. Baric, J., Bibi, R., Bohner, M., Nosheen, A., Pecaric, J.: Jensen Inequalities and Their Applications on Time Scales. Element, Zagreb (2015)

5. Baric, J., Nosheen, A., Pečarić, J.: Time scale Hardy-type inequalities with general kernel for superquadratic functions. Proc. A. Razmadze Math. Inst. 165, 1-18 (2014)

6. Bibi, R., Nosheen, A., Pečarić, J.: Refinements of Hardy-type inequalities on time scales. Commun. Math. Anal. 17(1), 67-87 (2014)

7. Bohner, M., Matthews, T.: Ostrowski inequalities on time scales. J. Inequal. Pure Appl. Math. 9(1), Art. 6 (2008)

8. Bohner, M., Nosheen, A., Pečarić, J., Younas, A.: Some dynamic Hardy type inequalities on time scales. J. Math. Inequal. 8(1), 185-199 (2014)

9. Bohner, M., Peterson, A.: Dynamic Equations on Time Scales: An Introduction with Applications. Birkhäuser Boston, Boston (2001)

10. Bohner, M., Peterson, A.: Advances in Dynamic Equations on Time Scales. Birkhäuser, Boston (2003)

11. Donchev, T., Nosheen, A., Pečarić, J.: Hardy-type inequalities on time scales via convexity in several variables. ISRN Math. Anal. 2013, Article ID 903196 (2013) 
12. Dragomir, S.S., Cerone, P., Barnett, N.S., Roumeliotis, J.: An inequality of the Ostrowski type for double integrals and applications for cubature formulae. Tamsui Oxf. J. Math. Sci. 16(1), 1-16 (2000)

13. Khan, J., Khan, M.A., Pečarić, J.: On Jensen's type inequalities via generalized majorization inequalities. Filomat 32(16), 5719-5733 (2018)

14. Khan, K.A., Nosheen, A., Pečarić, J.: $n$-Exponential convexity of some dynamic Hardy-type functionals. J. Math. Inequal. 8(2), 331-347 (2014)

15. Khan, M.A., Khan, J., Pečarić, J.: Generalizations of Sherman's inequality by Montgomery identity and Green function. Mong. Math. J. 19, 46-63 (2015)

16. Khan, M.A., Khan, J., Pečarić, J.: Generalization of Jensen's and Jensen-Steffensen's inequalities by generalized majorization theorem. J. Math. Inequal. 11(4), 1049-1074 (2017)

17. Mitrinovic, D.S., Pecaric, J.E., Fink, A.M.: Inequalities for Functions and Their Integrals and Derivatives. Kluwer Academic, Dordrecht (1994)

18. Özkan, U.M., Yilidrim, H.: Grüss type inequalities for double integrals on time scales. Comput. Math. Appl. 57(3), 436-444 (2009)

19. Sheng, Q., Fadag, M., Henderson, J., Davis, J.M.: An exploration of combined dynamic derivatives on time scales and their applications. Nonlinear Anal., Real World Appl. 7(3), 395-413 (2006)

\section{Submit your manuscript to a SpringerOpen ${ }^{\circ}$ journal and benefit from:}

- Convenient online submission

- Rigorous peer review

Open access: articles freely available online

- High visibility within the field

- Retaining the copyright to your article

Submit your next manuscript at $\gg$ springeropen.com 